\title{
Discrete Wiener-Hopf operators on spaces with Muckenhoupt weight
}

by

\author{
A. BÖTTCHER and M. SEYBOLD (Chemnitz)
}

Abstract. The discrete Wiener-Hopf operator generated by a function $a\left(e^{i \theta}\right)$ with the Fourier series $\sum_{n \in \mathbb{Z}} a_{n} e^{i n \theta}$ is the operator $T(a)$ induced by the Toeplitz matrix $\left(a_{j-k}\right)_{j, k=0}^{\infty}$ on some weighted sequence space $\ell^{p}\left(\mathbb{Z}_{+}, w\right)$. We assume that $w$ satisfies the Muckenhoupt $A_{p}$ condition and that $a$ is a piecewise continuous function subject to some natural multiplier condition. The last condition is in particular satisfied if $a$ is of bounded variation. Our main result is a Fredholm criterion and an index formula for $T(a)$. It implies that the essential spectrum of $T(a)$ results from the essential range of $a$ by filling in certain horns between the endpoints of each jump. The shape of these horns is determined by the indices of powerlikeness of the weight $w$.

1. Introduction. The theories of discrete Wiener-Hopf operators on $\ell^{2}\left(\mathbb{Z}_{+}\right)$, of Wiener-Hopf integral operators on $L^{2}\left(\mathbb{R}_{+}\right)$, and of Toeplitz operators on the Hardy space $H^{2}(\mathbb{T})$ are equivalent. The situation trifurcates dramatically when changing the exponent 2 to an exponent $p \in(1, \infty)$ or when equipping the spaces with a (Muckenhoupt) weight. Then we encounter three different theories, each with its own difficulties and techniques, although, and this is something like a miracle, the final results are almost the same.

We here consider operators with piecewise continuous symbols. The Fredholm theory of Toeplitz operators with piecewise continuous symbols on $H^{p}(\mathbb{T}, \varrho)$ with a power weight $\varrho$ has its roots in the work of Muskhelishvili, Gakhov, Khvedelidze, Simonenko, Widom, Devinatz, Shamir, to mention only a few principal figures, and it was raised to an impressive and beautiful edifice by Gohberg and Krupnik in the late sixties and early seventies (see [11] and the references therein). In the late eighties, Spitkovsky [17] completed the Fredholm theory of Toeplitz operators with piecewise continuous symbols on $H^{p}(\mathbb{T}, \varrho)$ in the case where $\varrho$ is an arbitrary Muckenhoupt weight. Subsequently, Karlovich and one of the authors were able to accomplish the corresponding theory for the spaces $H^{p}(\Gamma, \varrho)$ where $\Gamma$ is an arbi-

2000 Mathematics Subject Classification: Primary 47B35. 
trary Carleson (= Ahlfors-David) curve and $\varrho$ is an arbitrary Muckenhoupt weight (see [1]). The circular arcs prevailing in the Gohberg-Krupnik theory metamorphose into logarithmic spirals and eventually into logarithmic leaves with a halo in the case of general curves and weights. A key technique in Toeplitz theory is Wiener-Hopf factorization (in the sense of [16]).

Wiener-Hopf factorization is not applicable to Wiener-Hopf operators on the spaces $\ell^{p}\left(\mathbb{Z}_{+}, w\right)$ and $L^{p}\left(\mathbb{R}_{+}, w\right)$. The pioneering work on such operators was done by Duduchava [6]-[9] in the seventies. He established a round Fredholm theory for both discrete and integral Wiener-Hopf operators with piecewise continuous symbols on $\ell^{p}\left(\mathbb{Z}_{+},(n+1)^{\lambda}\right)$ and $L^{p}\left(\mathbb{R}_{+}\right)$, and he elaborated the fundamentals of the techniques that are used up to the present (and also in this paper). Schneider [15] extended the results to $L^{p}$ spaces with arbitrary power weights. The books [9] and [14] are excellent expositions of this topic (see also [3]).

Wiener-Hopf integral operators with piecewise continuous symbols were studied on $L^{p}\left(\mathbb{R}_{+}, w\right)$ with a Muckenhoupt weight $w$ by Spitkovsky and one of the authors in [4]. The approach of [4] is as follows: localization techniques reduce the problems to Wiener-Hopf operators with piecewise constant symbols, these can be interpreted as singular integral operators on $L^{p}\left(\mathbb{R}_{+}, w\right)$, and the latter operators can be transformed into Toeplitz operators on $H^{p}(\mathbb{T}, \varrho)$.

In the present paper we consider discrete Wiener-Hopf operators with piecewise continuous symbols on $\ell^{p}\left(\mathbb{Z}_{+}, w\right)$ in the case where $w$ is an arbitrary Muckenhoupt weight. Neither Wiener-Hopf factorization nor the strategy mentioned in the previous paragraph is applicable to such operam tors. We will rather employ an ingenious formula of Duduchava [8] for the inverse of $T\left(\varphi_{\alpha}\right)$ where $\varphi_{\alpha}$ is a certain canonical piecewise continuous function and will have recourse to some recent techniques developed in [1] to tackle general Muckenhoupt weights.

Duduchava and Schneider showed that the essential spectra of WienerHopf operators on $\ell^{p}\left(\mathbb{Z}_{+}, w\right)$ and $L^{p}\left(\mathbb{R}_{+}, w\right)$ contain certain circular arcs if $w$ is a power weight. In [4], it was observed that these circular arcs may blow up to horns for integral operators on $L^{p}\left(\mathbb{R}_{+}, w\right)$ if $w$ is a general Muckenhoupt weight. We here prove the emergence of horns for discrete operators on $\ell^{p}\left(\mathbb{Z}_{+}, w\right)$ with a Muckenhoupt weight $w$. As already said, although our techniques are (and must be) different from those used in the $L^{p}\left(\mathbb{R}_{+}, w\right)$ and $H^{p}(\mathbb{T}, \varrho)$ settings, the final results do all coincide in an astonishing manner.

The paper is organized as follows. Section 2 contains the main results, while Sections 3 to 6 are devoted to the proofs. Sections 3 and 4 deal with some properties of Muckenhoupt weights, in Section 5 we consider discrete Wiener-Hopf operators with certain canonical symbols, and in Section 6 we employ localization techniques to extend the results of Section 5 to more general piecewise continuous symbols. In Section 7 we establish an invertibility criterion.

2. Main results. Throughout this paper we assume that $1<p<\infty$. A weight on $\mathbb{Z}_{+}=\{0,1, \ldots\}$ is a sequence $w=\left\{w_{n}\right\}_{n=0}^{\infty}$ of positive numbers. Given a weight $w$, we denote by $\ell^{p}(w):=\ell^{p}\left(\mathbb{Z}_{+}, w\right)$ the Banach space of all complex-valued sequences $x=\left\{x_{n}\right\}_{n=0}^{\infty}$ such that

$$
\|x\|_{\ell^{p}(w)}:=\left(\sum_{n=0}^{\infty}\left|x_{n}\right|^{p} w_{n}^{p}\right)^{1 / p}<\infty .
$$

We write $w \in A_{p}$ and say that $w$ is a Muckenhoupt weight for $\ell^{p}$ if there is a constant $C<\infty$ such that

$$
\frac{1}{n-m+1}\left(\sum_{k=m}^{n} w_{k}^{p}\right)^{1 / p}\left(\sum_{k=m}^{n} w_{k}^{-q}\right)^{1 / q}<C
$$

for all $m, n$ with $0 \leq m \leq n$; here and in what follows, $q$ is given by $1 / p+$ $1 / q=1$. Hunt, Muckenhoupt, and Wheeden [12] showed that the matrix

$$
S_{+}:=\left(\begin{array}{ccccc}
0 & -1 & -1 / 2 & -1 / 3 & \ldots \\
1 & 0 & -1 & -1 / 2 & \ldots \\
1 / 2 & 1 & 0 & -1 & \ldots \\
1 / 3 & 1 / 2 & 1 & 0 & \ldots \\
\cdots & \ldots & \ldots & \ldots & \ldots
\end{array}\right)
$$

generates a bounded operator on $\ell^{p}(w)$ if and only if $w \in A_{p}$.

Weights of the form $\left\{(n+1)^{\lambda}\right\}_{n=0}^{\infty}$ are referred to as power weights. It is readily seen that a power weight belongs to $A_{p}$ if and only if $-1 / p<\lambda<1 / q$. Given a weight $w=\left\{w_{n}\right\}_{n=0}^{\infty} \in A_{p}$, we define

$$
I(w)=\left\{\lambda \in \mathbb{R}:\left\{w_{n}(n+1)^{\lambda}\right\}_{n=0}^{\infty} \in A_{p}\right\} .
$$

THEOREM 2.1. For every $w \in A_{p}$, the set $I(w)$ is an open interval that contains the origin and whose length is at most 1.

Thus, we can write $I(w)=(-\mu, 1-\nu)$ with certain numbers $\mu=\mu(w)$ and $\nu=\nu(w)$ satisfying $0<\mu \leq \nu<1$. We call $\mu$ and $\nu$ the indices of powerlikeness of $w$. Notice that if $w_{n}=(n+1)^{\lambda}$, then $\mu=\nu=1 / p+\lambda$ (which indicates that it might be more appropriate to call $\mu-1 / p$ and $\nu-1 / p$ the indices of powerlikeness; however, we here adopt the terminology introduced in [1]). In a sense, the larger the gap between $\mu$ and $\nu$ is, the more a weight differs from a power weight. The following result shows that there exist Muckenhoupt weights with arbitrarily prescribed indices of powerlikeness. 
THEOREM 2.2. Let $\lambda, \varepsilon, \eta$ be real numbers and suppose $\eta \neq 0$. The weight $w$ given by

$$
w_{n}=(n+e)^{\lambda+\varepsilon \sin (\eta \log (\log (n+e)))}
$$

belongs to $A_{p}$ if and only if

$$
-1 / p<\lambda-|\varepsilon| \sqrt{1+\eta^{2}} \leq \lambda+|\varepsilon| \sqrt{1+\eta^{2}}<1 / q .
$$

The indices of powerlikeness of $w$ are

$$
\mu=1 / p+\lambda-|\varepsilon| \sqrt{1+\eta^{2}}, \quad \nu=1 / p+\lambda+|\varepsilon| \sqrt{1+\eta^{2}} .
$$

In particular, given any numbers $\mu, \nu$ satisfying $0<\mu \leq \nu<1$, there exists a weight of the form (3) such that (4) and (5) hold.

Given a weight $w=\left\{w_{n}\right\}_{n=0}^{\infty}$ on $\mathbb{Z}_{+}$, we denote by $\ell^{p}(\mathbb{Z}, w)$ the Banach space of all complex-valued sequences $x=\left\{x_{n}\right\}_{n=-\infty}^{\infty}$ for which

$$
\|x\|_{\ell^{p}(\mathbb{Z}, w)}:=\left(\sum_{n=-\infty}^{\infty}\left|x_{n}\right|^{p} w_{|n|}^{p}\right)^{1 / p}<\infty ;
$$

thus, we identify $w$ with its continuation to an even weight on all of $\mathbb{Z}$.

If $X$ and $Y$ are Banach spaces, we let $\mathcal{L}(X, Y)$ and $\mathcal{K}(X, Y)$ denote the bounded and compact linear operators from $X$ to $Y$, respectively. As usual, we put $\mathcal{L}(X, X)=: \mathcal{L}(X), \mathcal{K}(X, X)=: \mathcal{K}(X)$.

Let $\mathbb{T}$ be the complex unit circle. For $a \in L^{\infty}:=L^{\infty}(\mathbb{T})$, we denote by $\left\{a_{n}\right\}_{n=-\infty}^{\infty}$ the sequence of the Fourier coefficients of $a$,

$$
a_{n}=\frac{1}{2 \pi} \int_{0}^{2 \pi} a\left(e^{i \pi}\right) e^{-i n \theta} d \theta
$$

and we let $L(a)$ and $T(a)$ stand for the Laurent and Toeplitz matrices generated by $a$ :

$$
L(a)=\left(a_{j-k}\right)_{j, k=-\infty}^{\infty}, \quad T(a)=\left(a_{j-k}\right)_{j, k=0}^{\infty} .
$$

It is well known that $L(a)$ generates a bounded operator on $\ell^{2}(\mathbb{Z})$. We let $M_{p, w}$ denote the set of all $a \in L^{\infty}$ for which there exists a constant $C_{p, w, a}<\infty$ such that

$$
\|L(a) x\|_{\ell^{p}(\mathbb{Z}, w)} \leq C_{p, w, a}\|x\|_{\ell^{p}(\mathbb{Z}, w)} \quad \text { for all } x \in \ell^{2}(\mathbb{Z}) \cap \ell^{p}(\mathbb{Z}, w) .
$$

Clearly, if $a \in M_{p, w}$, then $L(a)$ and also $T(a)$, the compression of $L(a)$ to $\ell^{p}(w)$, induce bounded operators on $\ell^{p}(\mathbb{Z}, w)$ and $\ell^{p}(w)$, respectively. The operator $T(a)$ is referred to as the discrete Wiener-Hopf operator with the symbol $a$. The set $M_{p, w}$ is a Banach algebra with pointwise algebraic operations and the norm

$$
\|a\|_{p, w}:=\|L(a)\|_{\mathcal{L}\left(\ell^{p}(\mathbb{Z}, w)\right)}
$$

In the case where $w_{n}=1$ for all $n$, we abbreviate $\ell^{p}(\mathbb{Z}, w), \ell^{p}(w), M_{p, w},\|\cdot\|_{p, w}$ to $\ell^{p}(\mathbb{Z}), \ell^{p}, M_{p},\|\cdot\|_{p}$, respectively. Finally, we remark that $\|a\|_{\infty}$ always denotes the $L^{\infty}$ norm of $a$; since $\|L(a)\|_{\mathcal{L}\left(\ell^{2}(\mathbb{Z})\right)}=\|a\|_{\infty},(6)$ for $\ell^{2}(\mathbb{Z})$ gives the equality $\|a\|_{2}=\|a\|_{\infty}$.

A function $a: \mathbb{T} \rightarrow \mathbb{C}$ is said to be of bounded variation, $a \in \mathrm{BV}$, if the function $\tilde{a}:[0,2 \pi] \rightarrow \mathbb{C}, \theta \mapsto a\left(e^{i \theta}\right)$ is of bounded variation, that is, $\mathrm{V}(a):=\sup \sum_{j=1}^{n}\left|a\left(e^{i \theta_{j+1}}\right)-a\left(e^{i \theta_{j}}\right)\right|<\infty$, the supremum taken over all partitions $0 \leq \theta_{1}<\theta_{2}<\ldots<\theta_{n+1} \leq 2 \pi$.

Let PC stand for the closed algebra of all bounded piecewise continuous functions of $\mathbb{T}$ into $\mathbb{C}$. Thus, $a \in \mathrm{PC}$ if and only if $a$ is bounded and has finite one-sided limits $a(t \pm 0):=\lim _{\varepsilon \rightarrow 0 \pm 0} a\left(t e^{i \varepsilon}\right)$ at every point $t \in \mathbb{T}$. It is easily seen that $B V \subset P C$.

The Stechkin inequality states that if $w \in A_{p}$, then there is a constant $C_{p, w}<\infty$ depending only on $p$ and $w$ such that

$$
\|a\|_{p, w} \leq C_{p, w}\left(\|a\|_{\infty}+\mathrm{V}(a)\right)
$$

for all $a \in \mathrm{BV}$. In particular, BV $\subset M_{p, w}$. For spaces without weight, this inequality goes back to Stechkin [18]. A full proof for the case of general Muckenhoupt weights is in [2].

The algebra $M_{p, w}$ is continuously embedded in $L^{\infty}$ :

$$
\|a\|_{\infty} \leq\|a\|_{p, w} \quad \text { for all } a \in M_{p, w} .
$$

Indeed, taking into account that the adjoint of $L(a)$ on $\ell^{p}(\mathbb{Z}, w)$ is the operator $L(\bar{a})$ on $\ell^{q}\left(\mathbb{Z}, w^{-1}\right)$, and defining the operator $V$ by $(V f)_{n}:=\bar{f}_{-n}$, we get

(9) $\quad\|L(a)\|_{\mathcal{L}\left(\ell^{p}(\mathbb{Z}, w)\right)}=\|L(\bar{a})\|_{\mathcal{L}\left(\ell q\left(\mathbb{Z}, w^{-1}\right)\right)}=\|V L(\bar{a}) V\|_{\mathcal{L}\left(\ell^{q}\left(\mathbb{Z}, w^{-1}\right)\right)}$

$$
=\|L(a)\|_{\mathcal{L}\left(\ell q\left(\mathbb{Z}, w^{-1}\right)\right)}
$$

whence, by the Stein-Weiss interpolation theorem,

$$
\begin{aligned}
\|a\|_{\infty} & =\|L(a)\|_{\mathcal{L}\left(\ell^{2}(\mathbb{Z})\right)} \leq\|L(a)\|_{\mathcal{L}\left(\ell^{p}(\mathbb{Z}, w)\right)}^{1 / 2}\|L(a)\|_{\mathcal{L}\left(\ell^{q}\left(\mathbb{Z}, w^{-1}\right)\right)}^{1 / 2} \\
& =\|L(a)\|_{\mathcal{L}\left(\ell^{p}(\mathbb{Z}, w)\right)}^{1 / 2}\|L(a)\|_{\mathcal{L}\left(\ell^{p}(\mathbb{Z}, w)\right)}^{1 / 2}=\|a\|_{p, w} .
\end{aligned}
$$

Denote by $\mathrm{PC}_{p, w}$ the closure of $\mathrm{BV}$ in $M_{p, w}$. Estimate (8) implies that $\mathrm{PC}_{p, w} \subset \mathrm{PC}$, i.e., functions in $\mathrm{PC}_{p, w}$ have finite one-sided limits everywhere on $\mathbb{T}$. Since $M_{2}=L^{\infty}$, we have $\mathrm{PC}_{2}=\mathrm{PC}$.

Given a number $\delta \in(0,1)$ and two distinct points $z, w \in \mathbb{C}$, we put

$$
\mathcal{A}(z, w ; \delta)=\left\{\zeta \in \mathbb{C} \backslash\{z, w\}: \arg \frac{\zeta-w}{\zeta-z} \in 2 \pi \delta+2 \pi \mathbb{Z}\right\} \cup\{z, w\} .
$$

A moment's thought reveals that $\mathcal{A}(z, w ; \delta)$ is a circular arc between $z$ and $w$ that lies on the left (right, respectively) of the straight line passing through first $z$ and then $w$ if $0<\delta<1 / 2(1 / 2<\delta<1$, respectively). For $\delta=1 / 2$, 
$\mathcal{A}(z, w ; \delta)$ is nothing but the line segment $[z, w]$. Notice that for arbitrary $\delta \in(0,1)$ the line segment $[z, w]$ is seen at the angle $\min \{2 \pi \delta, 2 \pi(1-\delta)\}$ at the points of $\mathcal{A}(z, w, \delta)$. Given real numbers $\mu, \nu$ satisfying $0<\mu \leq \nu<1$, we define the horn $\mathcal{H}(z, w ; \mu, \nu)$ by

$$
\mathcal{H}(z, w ; \mu, \nu)=\bigcup_{\delta \in[\mu, \nu]} \mathcal{A}(z, w ; \delta) .
$$

To cover the case $z=w$, we finally put $\mathcal{H}(z, z ; \mu, \nu)=\{z\}$.

Let $0<\mu \leq \nu<1$. For $a \in \mathrm{PC}$, the set

$$
a_{\mu, \nu}:=\bigcup_{t \in \mathbb{T}} \mathcal{H}(a(t-0), a(t+0) ; \mu, \nu)
$$

results from the (essential) range of $a$ by filling in a well defined horn between the endpoints of each jump. For every $\delta \in[\mu, \nu]$, the set $a_{\delta, \delta}$ is a closed continuous curve entirely contained in $a_{\mu, \nu}$. The counter-clockwise orientation of $\mathbb{T}$ induces a natural orientation of the curves $a_{\delta, \delta}$. If $0 \notin a_{\mu, \nu}$, then $0 \notin a_{\delta, \delta}$ for every $\delta \in[\mu, \nu]$, and the winding number of $a_{\delta, \delta}$ about the origin is well defined and independent of $\delta \in[\mu, \nu]$. We denote this winding number by wind $a_{\mu, \nu}$.

An operator $A \in \mathcal{L}(X)$ is said to be Fredholm if it is invertible modulo compact operators. In that case the kernel and the cokernel dimensions of $A$ are finite, and their difference is called the index of $A$ and denoted by Ind $A$.
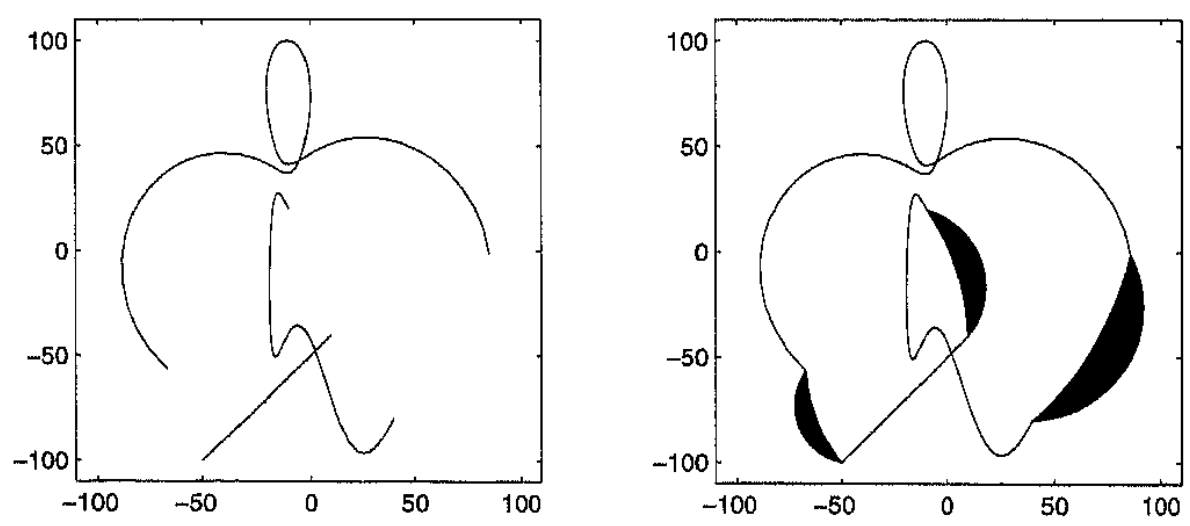

The range of some $a \in \mathrm{PC}_{p, w}$ vs. the essential spectrum of $T(a)$ in $\mathcal{L}\left(\ell^{p}(w)\right)$. The indices of powerlikeness of $w$ determine the shape of the horns.

Here is our main result.

THEOREM 2.3. Let $w \in A_{p}$ and let $\mu \leq \nu$ be the indices of powerlikeness of $w$. Let further $a \in \mathrm{PC}_{p, w}$. The operator $T(a)$ is Fredholm on $\ell^{p}(w)$ if and only if $0 \notin a_{\mu, \nu}$. In that case

$$
\operatorname{Ind} T(a)=- \text { wind } a_{\mu, \nu}
$$

For the power weights $w=\left\{(n+1)^{\lambda}\right\}_{n=0}^{\infty}$, this is Duduchava's theorem [6], [8]. Note that in this case $\mu=\nu=1 / p+\lambda$ and therefore the horns degenerate to circular arcs.

The following result clears up the matter of invertibility.

THEOREM 2.4. Let $w \in A_{p}$ and $a \in \mathrm{PC}_{p, w}$. If $T(a)$ is Fredholm of index $\kappa$ on $\ell^{p}(w)$, then $\operatorname{dim} \operatorname{Ker} T(a)=\max \{\kappa, 0\}$ and $\operatorname{dim} \operatorname{Coker} T(a)=$ $\max \{-\kappa, 0\}$. In particular, the operator $T(a)$ is invertible if and only if it is Fredholm of index zero.

3. Indices of powerlikeness. In this section we prove Theorem 2.1. To do this, we need the following theorem, which states an important property of Muckenhoupt weights.

THEOREM 3.1. If $w \in A_{p}$, then there is an $\varepsilon=\varepsilon_{p, w}>0$ such that $w^{r} \in A_{p}$ for all $r \in(1-\varepsilon, 1+\varepsilon)$.

This result is well known. Strömberg and Torchinsky [19, p. 11] state such a result in an abstract context. A full and self-contained proof is also in [2]; that proof is an appropriate modification of the very clear proof given by García-Cuerva and Rubio de Francia [10] for Muckenhoupt weights on $\mathbb{R}^{n}$.

To prove Theorem 2.1, notice first that 0 clearly lies in $I(w)$. Stein-Weiss interpolation shows that $I(w)$ is connected, and Theorem 3.1 implies that $I(w)$ is open. We are left with proving that the length of $I(w)$ is at most 1 . Assume the contrary, that is, assume there is a $\lambda \in \mathbb{R}$ such that

$$
\left\{v_{n}\right\}:=\left\{w_{n}(n+1)^{\lambda}\right\} \in A_{p}, \quad\left\{v_{n}(n+1)\right\}=\left\{w_{n}(n+1)^{\lambda+1}\right\} \in A_{p}
$$

Using the Muckenhoupt inequalities (1) for $\left\{v_{n}\right\}$ and $\left\{v_{n}(n+1)\right\}$ and taking into account Hölder's inequality, we obtain

$$
\begin{aligned}
\infty & >C^{2} \\
& \geq \frac{1}{n^{2}}\left(\sum_{k=0}^{n} v_{k}^{p}\right)^{1 / p}\left(\sum_{k=0}^{n} v_{k}^{-q}\right)^{1 / q}\left(\sum_{k=0}^{n}(k+1)^{p} v_{k}^{p}\right)^{1 / p}\left(\sum_{k=0}^{n}(k+1)^{-q} v_{k}^{-q}\right)^{1 / q} \\
& =\frac{1}{n^{2}}\left(\sum_{k=0}^{n} v_{k}^{p}\right)^{1 / p}\left(\sum_{k=0}^{n}(k+1)^{-q} v_{k}^{-q}\right)^{1 / q}\left(\sum_{k=0}^{n}(k+1)^{p} v_{k}^{p}\right)^{1 / p}\left(\sum_{k=0}^{n} v_{k}^{-q}\right)^{1 / q} \\
& \geq \frac{1}{n^{2}}\left(\sum_{k=0}^{n} \frac{1}{k+1}\right)\left(\sum_{k=0}^{n}(k+1)\right)=\frac{1}{n^{2}} \frac{(n+1)(n+2)}{2} \sum_{k=0}^{n} \frac{1}{k+1},
\end{aligned}
$$

which is a contradiction. 
4. Horns of prescribed shape. This section is devoted to the proof of Theorem 2.2.

Let $\Gamma$ stand for the half-line $\mathbb{R}_{+}=(0, \infty)$ or for the upper half-circle $\mathbb{T}_{+}=\{t \in \mathbb{C}:|t|=1, \operatorname{Im} t>0\}$. Let further $v: \Gamma \rightarrow(0, \infty)$ be a continuous function. We write $v \in A_{p}(T)$ if

$$
\sup _{\tau \in \Gamma \in>0} \frac{1}{\varepsilon}\left(\int_{\Gamma(\tau, \varepsilon)} v(t)^{p}|d t|\right)^{1 / p}\left(\int_{\Gamma(\tau, \varepsilon)} v(t)^{-q}|d t|\right)^{1 / q}<\infty,
$$

where $\Gamma(\tau, \varepsilon):=\{t \in \Gamma:|t-\tau|<\varepsilon\}$.

Throughout this section we assume that $w:[0, \infty) \rightarrow(0, \infty)$ is a function of the form

$$
w(x)=\exp F(x), \quad F(x)=f(\log \log (x+e)) \log (x+e)
$$

where $f \in C[0, \infty) \cap C^{1}[0, \infty)$ and $f$ and $f^{\prime}$ are bounded on $(0, \infty)$.

For $x \in \mathbb{R}$, let $[x]$ be the largest integer that is not greater than $x$, let $J_{x}$ be the segment $[[x],[x]+1]$, and define

$$
w_{0}(x)=\min _{y \in J_{x}} w(y), \quad w_{1}(x)=\max _{y \in J_{x}} w(y) .
$$

LEMMA 4.1. There is a constant $C<\infty$ depending only on $w$ such that

$$
w_{1}(x) / w_{0}(x) \leq C \quad \text { for all } x \in[0, \infty) .
$$

Proof. For every $x \in[0, \infty)$, there are $\alpha, \beta, \gamma \in J_{x}$ such that

$$
w_{0}(x)=w(\alpha), \quad w_{1}(x)=w(\beta), \quad w(\alpha)=w(\beta)+w^{\prime}(\gamma)(\alpha-\beta) .
$$

Consequently,

$$
\begin{aligned}
\frac{w_{0}(x)}{w_{1}(x)} & =\frac{w(\alpha)}{w(\beta)}=\frac{w(\beta)+w^{\prime}(\gamma)(\alpha-\beta)}{w(\beta)} \geq 1-\max _{y \in J_{x}} \frac{\left|w^{\prime}(y)\right|}{w(\beta)} \\
& =1-\max _{y \in J_{x}}\left|\frac{w(y)}{w(\beta)}\left(\frac{f^{\prime}(\log \log (y+e))}{y+e}+\frac{f(\log \log (y+e))}{y+e}\right)\right| \\
& \geq 1-\frac{\left\|f^{\prime}\right\|_{\infty}}{[x]+e}-\frac{\|f\|_{\infty}}{[x]+e} \geq \frac{1}{3}
\end{aligned}
$$

for all sufficiently large $x$, say for $x>N$. The assertion now follows with

$$
C:=\max \left\{3, \frac{w_{1}(0)}{w_{0}(0)}, \frac{w_{1}(1)}{w_{0}(1)}, \ldots, \frac{w_{1}(N)}{w_{0}(N)}\right\}
$$

The following lemma allows us to pass from $\mathbb{Z}_{+}$to $\mathbb{R}_{+}$.

LEMMA 4.2. We have

$$
\{w(n)\}_{n=0}^{\infty} \in A_{p} \Leftrightarrow w \in A_{p}\left(\mathbb{R}_{+}\right) .
$$

Proof. Let $m, n \in \mathbb{Z}_{+}$and $m \leq n$. With $C$ from Lemma 4.1 we get

$$
\begin{aligned}
\frac{1}{n-m+1} & \left(\sum_{k=m}^{n} w(k)^{p}\right)^{1 / p}\left(\sum_{k=m}^{n} w(k)^{-q}\right)^{1 / q} \\
& \leq \frac{1}{n-m+1}\left(\sum_{k=m}^{n} w_{1}(k)^{p}\right)^{1 / p}\left(\sum_{k=m}^{n} w_{0}(k)^{-q}\right)^{1 / q} \\
& =\frac{1}{n-m+1}\left(\int_{m}^{n+1} w_{1}(x)^{p} d x\right)^{1 / p}\left(\int_{m}^{n+1} w_{0}(x)^{-q} d x\right)^{1 / q} \\
& \leq \frac{1}{n-m+1}\left(\int_{m}^{n+1} C^{p} w_{0}(x)^{p} d x\right)^{1 / p}\left(\int_{m}^{n+1} C^{q} w_{1}(x)^{-q} d x\right)^{1 / q} \\
& \leq \frac{C^{2}}{n-m+1}\left(\int_{m}^{n+1} w(x)^{p} d x\right)^{1 / p}\left(\int_{m}^{n+1} w(x)^{-q} d x\right)^{1 / q} .
\end{aligned}
$$

Hence, if $w \in A_{p}\left(\mathbb{R}_{+}\right)$then $\{w(n)\} \in A_{p}$.

In order to prove the reverse implication, let $a, b \in \mathbb{R}_{+}$and $a<b$. If $a<b<a+1$ and $[a]=[b]$, we have

$$
\begin{aligned}
\frac{1}{b-a}\left(\int_{a}^{b} w(x)^{p} d x\right)^{1 / p} & \left(\int_{a}^{b} w(x)^{-q} d x\right)^{1 / q} \\
& \leq \frac{1}{b-a}(b-a)^{1 / p} w_{1}([a])(b-a)^{1 / q} \frac{1}{w_{0}([a])} \leq C .
\end{aligned}
$$

If $a<b<a+1$ and $[a]+1=[b]$, then $w_{0}([a]) \leq w([b]) \leq w_{1}([a])$, whence

$$
\begin{aligned}
& \frac{1}{b-a}\left(\int_{a}^{b} w(x)^{p} d x\right)^{1 / p}\left(\int_{a}^{b} w(x)^{-q} d x\right)^{1 / q} \\
& \quad \leq \frac{\max \left\{w_{1}([a]), w_{1}([b])\right\}}{\min \left\{w_{0}([a]), w_{0}([b])\right\}} \\
& \quad \leq \frac{\max \left\{C w_{0}([a]), C w_{0}([b])\right\}}{w([b])} \frac{w([b])}{\min \left\{C^{-1} w_{1}([a]), C^{-1} w_{1}([b])\right\}} \leq C^{2}
\end{aligned}
$$

Finally, if $a+1 \leq b$ then

$$
\frac{[b]-[a]+1}{b-a}<\frac{b+1-a+1}{b-a}=1+\frac{2}{b-a} \leq 3
$$

and thus 


$$
\begin{aligned}
\frac{1}{b-a}\left(\int_{a}^{b} w(x)^{p} d x\right)^{1 / p}\left(\int_{a}^{b} w(x)^{-q} d x\right)^{1 / q} \\
\leq \frac{1}{b-a}\left(\int_{[a]}^{[b]+1} w_{1}(x)^{p} d x\right)^{1 / p}\left(\int_{[a]}^{[b]+1} w_{0}(x)^{-q} d x\right)^{1 / q} \\
=\frac{1}{b-a}\left(\sum_{k=[a]}^{[b]} w_{1}(k)^{p}\right)^{1 / p}\left(\sum_{k=[a]}^{[b]} w_{0}(k)^{-q}\right)^{1 / q} \\
\leq \frac{C^{2}}{b-a}\left(\sum_{k=[a]}^{[b]} w(k)^{p}\right)^{1 / p}\left(\sum_{k=[a]}^{[b]} w(k)^{-q}\right)^{1 / q} \\
\leq \frac{3 C^{2}}{[b]-[a]+1}\left(\sum_{k=[a]}^{[b]} w(k)^{p}\right)^{1 / p}\left(\sum_{k=[a]}^{[b]} w(k)^{-q}\right)^{1 / q}
\end{aligned}
$$

This shows that $w \in A_{p}\left(\mathbb{R}_{+}\right)$whenever $\{w(n)\} \in A_{p}$.

We now pass to the half-circle $\mathbb{T}_{+}$.

Lemma 4.3. Define $\varrho: \mathbb{T}_{+} \rightarrow(0, \infty)$ by

$$
\varrho(t)=w\left(i \frac{t+1}{t-1}\right)|t-1|^{1-2 / p} .
$$

Then

$$
w \in A_{p}\left(\mathbb{R}_{+}\right) \Leftrightarrow \varrho \in A_{p}\left(\mathbb{T}_{+}\right) .
$$

This is a standard result. A proof is in [4, pp. 265-266], for example.

Muckenhoupt weights on bounded simple Carleson curves can be studied with the help of the machinery developed in [1]. The following theorem is an immediate consequence of Theorems 1.13 and 2.33 and Lemma 2.35 of [1].

THEOREM 4.4. Suppose $\varrho: \mathbb{T}_{+} \rightarrow(0, \infty)$ is a weight of the form

$$
\varrho(t)=e^{G(|t-1|)}, \quad t \in \mathbb{T}_{+},
$$

with a function $G \in C(0,2] \cap C^{1}(0,2]$ such that $r G^{\prime}(r)$ is bounded for $r$ in $(0,2]$. Then

(a) the upper limit $\Phi_{\varrho}(x):=\lim \sup _{r \rightarrow 0}(G(x r)-G(r))$ is finite for each $x \in(0, \infty)$;

(b) the limits $\alpha(\varrho):=\lim _{x \rightarrow 0}\left(\Phi_{\varrho}(x) / \log x\right), \beta(\varrho):=\lim _{x \rightarrow \infty}\left(\Phi_{\varrho}(x) / \log x\right)$ exist and $-\infty<\alpha(\varrho) \leq \beta(\varrho)<\infty$;

(c) we have

$$
\varrho \in A_{p}\left(\mathbb{T}_{+}\right) \Leftrightarrow-1 / p<\alpha(\varrho) \leq \beta(\varrho)<1 / q .
$$

We apply Theorem 4.4 to the weight $\varrho$ introduced in Lemma 4.3. If $t \in \mathbb{T}_{+}$and $|t-1|=r$, then $i(t+1) /(t-1)=\sqrt{4 / r^{2}-1}$, and hence $\varrho(t)=$ $w\left(\sqrt{4 / r^{2}-1}\right) r^{1-2 / p}=e^{G(r)}$ with $G(r)=F\left(\sqrt{4 / r^{2}-1}\right)+(1-2 / p) \log r$.

LEMMA 4.5. We have

$$
\Phi_{\varrho}(x)=\limsup _{R \rightarrow \infty}(F(R / x)-F(R))+(1-2 / p) \log x .
$$

Proof. Obviously,

$$
\begin{aligned}
\Phi_{Q}(x) & =\limsup _{r \rightarrow 0}\left(F\left(\sqrt{4 /(x r)^{2}-1}\right)-F\left(\sqrt{4 / r^{2}-1}\right)\right)+(1-2 / p) \log x \\
& =\limsup _{R \rightarrow \infty}\left(F\left(\sqrt{R^{2} / x^{2}-1}\right)-F\left(\sqrt{R^{2}-1}\right)\right)+(1-2 / p) \log x,
\end{aligned}
$$

and since $F\left(\sqrt{R^{2} / x^{2}-1}\right)-F\left(\sqrt{R^{2}-1}\right)$ equals

$$
F\left(\sqrt{R^{2} / x^{2}-1}\right)-F(R / x)+F(R)-F\left(\sqrt{R^{2}-1}\right)+F(R / x)-F(R),
$$

it suffices to prove that

$$
\lim _{y \rightarrow \infty}\left(F\left(\sqrt{y^{2}-1}\right)-F(y)\right)=0 .
$$

Because

$$
F(y)-F\left(\sqrt{y^{2}-1}\right)=F^{\prime}(\xi)\left(y-\sqrt{y^{2}-1}\right)=\frac{F^{\prime}(\xi)}{y+\sqrt{y^{2}-1}}
$$

with $\sqrt{y^{2}-1} \leq \xi \leq y$ and

$$
\left|F^{\prime}(\xi)\right| \leq \frac{\left\|f^{\prime}\right\|_{\infty}}{\xi+e}+\frac{\|f\|_{\infty}}{\xi+e} \leq \frac{\left\|f^{\prime}\right\|_{\infty}+\|f\|_{\infty}}{y}
$$

it is clear that (11) holds.

LEMMA 4.6. If $f \in C^{2}(0, \infty)$ and $f, f^{\prime}, f^{\prime \prime}$ are bounded on $(0, \infty)$, then

$$
\limsup _{R \rightarrow \infty}(F(R / x)-F(R))= \begin{cases}-M(f) \log x & \text { for } x \in(0,1] \\ -m(f) \log x & \text { for } x \in[1, \infty),\end{cases}
$$

where

$$
m(f)=\liminf _{y \rightarrow \infty}\left(f(y)+f^{\prime}(y)\right), \quad M(f)=\limsup _{y \rightarrow \infty}\left(f(y)+f^{\prime}(y)\right) .
$$

This can be verified by the arguments of the proof of [1, Proposition 1.19]. Lemmas 4.5 and 4.6 yield that

$$
\alpha(\varrho)=-M(f)+1-2 / p, \quad \beta(\varrho)=-m(f)+1-2 / p .
$$

We therefore deduce from Theorem 4.4(c) that $\varrho \in A_{p}\left(\mathbb{T}_{+}\right)$if and only if

$$
-1 / p<-M(f)+1-2 / p \leq-m(f)+1-2 / p<1 / q,
$$

which is equivalent to the condition

$$
-1 / p<m(f) \leq M(f)<1 / q .
$$


LEMMA 4.7. If $f(x)=\lambda+\varepsilon \sin (\eta x)$ and $\eta \neq 0$, then

$$
m(f)=\lambda-|\varepsilon| \sqrt{1+\eta^{2}}, \quad M(f)=\lambda+|\varepsilon| \sqrt{1+\eta^{2}} .
$$

Thus, by (12) and Lemma 4.7, the weight $\varrho$ corresponding to $f(x)=$ $\lambda+\varepsilon \sin (\eta x)$ belongs to $A_{p}\left(\mathbb{T}_{+}\right)$if and only if (4) holds. Lemmas 4.2 and 4.3 complete the proof of Theorem 2.2.

5. Local representatives. In this section we prove Theorem 2.3 for a class of special symbols with a single jump.

For $\gamma \in \mathbb{C}$ and $\tau \in \mathbb{T}$, we define $\varphi_{\gamma, \tau}: \mathbb{T} \backslash\{\tau\} \rightarrow \mathbb{C}$ by

$$
\varphi_{\gamma, \tau}(t)=\exp (i \gamma \arg (-t / \tau))
$$

the argument taken in $(-\pi, \pi]$. It is easily seen that $\varphi_{\gamma, \tau}$ is $C^{\infty}$ on $\mathbb{T} \backslash\{\tau\}$ and that $\varphi_{\gamma, \tau}(\tau-0)=e^{\pi i \gamma}$ and $\varphi_{\gamma, \tau}(\tau+0)=e^{-\pi i \gamma}$. The Fourier coefficients of $\varphi_{\gamma, \tau}$ are

$$
\left(\varphi_{\gamma, \tau}\right)_{n}= \begin{cases}-\frac{\sin (\pi \gamma)}{\pi(n-\gamma)} \tau^{-n} & \text { for } n \neq \gamma \\ (-1)^{n} \tau^{-n} & \text { for } n=\gamma\end{cases}
$$

Also, for $\gamma \in \mathbb{C}$, we denote by $(1-z)^{\gamma}$ the branch given by

$$
(1-z)^{\gamma}=\exp (\gamma \log |1-z|+i \gamma \arg (1-z)), \quad \arg (1-z) \in(-\pi, \pi],
$$

we define $\xi_{\gamma, \tau}, \eta_{\gamma, \tau}: \mathbb{T} \backslash\{\tau\} \rightarrow \mathbb{C}$ by

$$
\xi_{\gamma, \tau}(t)=(1-\tau / t)^{\gamma}, \quad \eta_{\gamma, \tau}(t)=(1-t / \tau)^{\gamma},
$$

and we let $T\left(\xi_{\gamma, \tau}\right), T\left(\eta_{\gamma, \tau}\right)$ stand for the Toeplitz matrices

$$
T\left(\xi_{\gamma, \tau}\right)=\left(\left(\xi_{\gamma, \tau}\right)_{j-k}\right)_{j, k=0}^{\infty}, \quad T\left(\eta_{\gamma, \tau}\right)=\left(\left(\eta_{\gamma, \tau}\right)_{j-k}\right)_{j, k=0}^{\infty}
$$

with

$$
\left(\xi_{\gamma, \tau}\right)_{-n}=\left\{\begin{array}{ll}
(-\tau)^{n}\left(\begin{array}{l}
\gamma \\
n
\end{array}\right) & \text { for } n \geq 0, \\
0 & \text { for } n<0,
\end{array} \quad\left(\eta_{\gamma, \tau}\right)_{n}= \begin{cases}\left(-\frac{1}{\tau}\right)^{n}\left(\begin{array}{l}
\gamma \\
n
\end{array}\right) & \text { for } n \geq 0 \\
0 & \text { for } n<0\end{cases}\right.
$$

Notice that $\xi_{\gamma, \tau}$ and $\eta_{\gamma, \tau}$ belong to $L^{1}(\mathbb{T})$ if and only if $\operatorname{Re} \gamma>-1$, in which case the numbers $\left(\xi_{\gamma, \tau}\right)_{n}$ and $\left(\eta_{\gamma, \tau}\right)_{n}$ are the Fourier coefficients of these functions.

Finally, for $\gamma \in \mathbb{C}$, put

$$
M_{\gamma}=\operatorname{diag}\left(\mu_{n}^{(\gamma)}\right)_{n=0}^{\infty}, \quad \mu_{n}^{(\gamma)}=\left(\begin{array}{c}
\gamma+n \\
n
\end{array}\right) .
$$

It is not difficult to see that if $K$ is a compact subset of $\mathbb{C} \backslash\{-1,-2, \ldots\}$, then there is a constant $c_{K} \in(0, \infty)$ depending only on $K$ such that

$$
c_{K}^{-1}(n+1)^{\operatorname{Re} \gamma} \leq\left|\mu_{n}^{(\gamma)}\right| \leq c_{K}(n+1)^{\operatorname{Re} \gamma}
$$

for all $n \geq 0$ and all $\gamma \in K$ (see, e.g., [3, Lemma 6.21]).
In what follows, we think of $\tau \in \mathbb{T}$ as being fixed and therefore we abbreviate $\varphi_{\gamma, \tau}, \xi_{\gamma, \tau}, \eta_{\gamma, \tau}$ to $\varphi_{\gamma}, \xi_{\gamma}, \eta_{\gamma}$, respectively.

TheOREM 5.1 (Duduchava). If $\alpha \in \mathbb{C} \backslash \mathbb{Z}$, then

$$
T\left(\eta_{-\alpha}\right) T\left(\xi_{\alpha}\right)=\Gamma_{\alpha} M_{\alpha} T\left(\varphi_{-\alpha}\right) M_{-\alpha}, \quad \Gamma_{\alpha}:=\frac{\pi \alpha}{\sin (\pi \alpha)},
$$

in the sense that all entries of the infinite matrices are well defined and that corresponding entries are equal.

This theorem was established by Duduchava in [6], [8]. We remark that (15) is in fact a set of identities involving binomial coefficients. In [3, Theorem 6.20], this set of identities is proved by expanding a hypergeometric function into a power series in two different ways.

Corollary 5.2. Let $\alpha \in \mathbb{C} \backslash \mathbb{Z}$ and put

$$
A_{\alpha}=\Gamma_{\alpha} M_{\alpha} T\left(\varphi_{-\alpha}\right) M_{-\alpha} .
$$

We have $A_{\alpha} T\left(\varphi_{\alpha}\right)=I$ for $\operatorname{Re} \alpha>-1$ and $T\left(\varphi_{\alpha}\right) A_{\alpha}=I$ for $\operatorname{Re} \alpha<1$ in the sense that the entries of the infinite matrices are well defined and that corresponding entries coincide.

Proof. Suppose first that $\operatorname{Re} \alpha=0$. Then $\xi_{ \pm \alpha}$ and $\eta_{ \pm \alpha}$ belong to the Hardy spaces $\overline{H^{\infty}(\mathbb{T})}$ and $H^{\infty}(\mathbb{T})$, respectively. The matrices $T\left(\varphi_{ \pm \alpha}\right)$, $T\left(\xi_{ \pm \alpha}\right), T\left(\eta_{ \pm \alpha}\right), M_{ \pm \alpha}$ all induce bounded operators on $\ell^{2}$, and the identity $\varphi_{\alpha}=\xi_{-\alpha} \eta_{\alpha}$ implies that $T\left(\varphi_{\alpha}\right)=T\left(\xi_{-\alpha}\right) T\left(\eta_{\alpha}\right)$. This equality in conjunction with Theorem 5.1 gives

$$
T\left(\varphi_{\alpha}\right) A_{\alpha}=T\left(\xi_{-\alpha}\right) T\left(\eta_{\alpha}\right) T\left(\eta_{-\alpha}\right) T\left(\xi_{\alpha}\right)=I .
$$

In order to extend (17) to $\operatorname{Re} \alpha<1$, it suffices to prove that the entries of $T\left(\varphi_{\alpha}\right)$ are well defined analytic functions in $H:=\{\alpha \in \mathbb{C} \backslash \mathbb{Z}: \operatorname{Re} \alpha<1\}$. We have

$$
\left(T\left(\varphi_{\alpha}\right) A_{\alpha}\right)_{j k}=\sum_{n=0}^{\infty}\left(\varphi_{\alpha}\right)_{j-n} \Gamma_{\alpha} \mu_{n}^{(\alpha)}\left(\varphi_{-\alpha}\right)_{n-k} \mu_{k}^{(-\alpha)},
$$

and since $\left|\left(\varphi_{ \pm \alpha}\right)_{n}\right|=O(1 / n)$ and $\left|\mu_{n}^{(\alpha)}\right|=O\left(n^{\text {Re } \alpha}\right)$ uniformly with respect to compact subsets of $H$ (recall (13) and (14)), we get

$$
\left|\left(\varphi_{\alpha}\right)_{j-n} \mu_{n}^{(\alpha)}\left(\varphi_{-\alpha}\right)_{n-k}\right|=O\left(n^{\operatorname{Re} \alpha-2}\right)
$$

uniformly with respect to compact subsets of $H$. As $\operatorname{Re} \alpha-2<-1$ for $\alpha \in H$, it follows that the series on the right of (18) defines an analytic function. The case $\operatorname{Re} \alpha>-1$ can be treated analogously.

Corollary 5.2 shows that, at least for $-1<\operatorname{Re} \alpha<1, A_{\alpha}$ may be interpreted as a formal inverse of $T\left(\varphi_{\alpha}\right)$. In order to understand the conditions under which $A_{\alpha}$ is really the inverse of $T\left(\varphi_{\alpha}\right)$, we need to know when the operator $T\left(\varphi_{-\alpha}\right)$ is bounded on $\ell^{p}(w)$. By $(7)$, this is the case if $w \in A_{p}$. 
The following result shows that the converse is also true. Notice that by (13) the matrix $T\left(\varphi_{\gamma}\right)$ induces a bounded operator on $\ell^{p}(w)$ if and only if so does the matrix $T=\left(T_{k l}\right)_{k, l=0}^{\infty}$ with

$$
T_{k l}= \begin{cases}\frac{1}{k-l-\gamma} & \text { for } k-l-\gamma \neq 0 \\ 0 & \text { for } k-l-\gamma=0\end{cases}
$$

THEOREM 5.3. Let $\gamma \in \mathbb{C} \backslash \mathbb{Z}$. If the matrix given by (19) generates a bounded operator on $\ell^{p}(w)$, then $w \in A_{p}$.

Proof. For $\gamma=0$, this theorem was proved in [12]. Our proof is a modification of that proof.

Let $a=\operatorname{Re} \gamma, b=\operatorname{Im} \gamma$, and denote by $\bar{a}$ the smallest natural number which is greater than $|a|$. Fix $m, n \in \mathbb{Z}_{+}$so that $m \leq n$. If

$$
n+\bar{a} \leq k \leq 2 n+\bar{a}-m, \quad m \leq l \leq n,
$$

then

$$
\operatorname{Re}(k-l-\gamma)=k-l-a \geq k-l-|a|>k-l-\bar{a} \geq 0,
$$

whence $k-l-\gamma \neq 0$. Put

$$
x=\sum_{l=m}^{n} w_{l}^{-q} e_{l}, \quad A=\sum_{l=m}^{n} w_{l}^{-q}
$$

where $e_{l}$ is the $l$ th element of the standard basis of $\ell^{p}(w)$. We have

$$
\left|(T x)_{k}\right|=\left|\sum_{l=m}^{n} \frac{w_{l}^{-q}}{k-l-\gamma}\right| \geq \sum_{l=m}^{n} \operatorname{Re} \frac{w_{l}^{-q}}{k-l-\gamma}=\sum_{l=m}^{n} \frac{k-l-a}{(k-l-a)^{2}+b^{2}} w_{l}^{-q} .
$$

It is not difficult to see that there is a constant $D>0$ that depends only on $a$ and $b$ such that

$$
\frac{k-l-a}{(k-l-a)^{2}+b^{2}} \geq \frac{D}{k-l-a}
$$

for all $k, l$ subject to (20). Thus,

$$
\left|(T x)_{k}\right| \geq \sum_{l=m}^{n} \frac{D}{k-l-a} w_{l}^{-q} \stackrel{(20),(21)}{\geq} D A \frac{1}{2 n-2 m+\bar{a}-a} .
$$

Finally, one can show without difficulty that there exists a constant $E>0$ depending only on $a$ such that $D /(2 n-2 m+\bar{a}-a) \geq E /(n-m+1)$. This implies that

$$
\left|(T x)_{k}\right| \geq E A \frac{1}{n-m+1}
$$

If $T$ is bounded on $\ell^{p}(w)$, then there is a constant $C<\infty$ such that $\|T y\| \leq C\|y\|$ for all $y \in \ell^{p}(w)$. Consequently, given any $d>0$, we have

$$
\left(\sum_{k:\left|(T y)_{k}\right| \geq d} w_{k}^{p}\right)^{1 / p} \leq C d^{-1}\|y\| .
$$

Letting $d=E A /(n-m+1)$ and $y=x$ from (21), we deduce from (22) and (21) that

$$
\begin{aligned}
\left(\sum_{k=n+\bar{a}}^{2 n+\bar{a}-m} w_{k}^{p}\right)^{1 / p} & \leq\left(\sum_{k:\left|(T x)_{k}\right| \geq d} w_{k}^{p}\right)^{1 / p} \\
& \leq C \frac{n-m+1}{E A}\left(\sum_{l=m}^{n}\left(w_{l}^{-q}\right)^{p} w_{l}^{p}\right)^{1 / p} \\
& =C \frac{n-m+1}{E A}\left(\sum_{l=m}^{n} w_{l}^{-q}\right)^{1 / p} \\
& =C \frac{n-m+1}{E}\left(\sum_{l=m}^{n} w_{l}^{-q}\right)^{-1 / q}
\end{aligned}
$$

Analogously one can prove that

$$
\left(\sum_{l=m}^{n} w_{l}^{p}\right)^{1 / p} \leq C \frac{n-m+1}{F}\left(\sum_{l=n+\bar{a}}^{2 n+\bar{a}-m} w_{l}^{-q}\right)^{-1 / q}
$$

with some constant $F>0$ depending only on $\gamma$.

Combining (23) and (24) we obtain

$$
\begin{aligned}
\frac{1}{(n-m+1)^{2}}\left(\sum_{l=n+\bar{a}}^{2 n+\bar{a}-m} w_{l}^{p}\right)^{1 / p}( & \left.\sum_{l=n+\bar{a}}^{2 n+\bar{a}-m} w_{l}^{-q}\right)^{1 / q} \\
& \times\left(\sum_{l=m}^{n} w_{l}^{p}\right)^{1 / p}\left(\sum_{l=m}^{n} w_{l}^{-q}\right)^{1 / q} \leq \frac{C^{2}}{E F} .
\end{aligned}
$$

By Hölder's inequality,

$$
\left(\sum_{l=n+\bar{a}}^{2 n+\bar{a}-m} w_{l}^{p}\right)^{1 / p}\left(\sum_{l=n+\bar{a}}^{2 n+\bar{a}-m} w_{l}^{-q}\right)^{1 / q} \geq \sum_{l=n+\bar{a}}^{2 n+\bar{a}-m} 1=n-m+1 .
$$

Thus,

$$
\frac{1}{n-m+1}\left(\sum_{l=m}^{n} w_{l}^{p}\right)^{1 / p}\left(\sum_{l=m}^{n} w_{l}^{-q}\right)^{1 / q} \leq \frac{C^{2}}{E F} .
$$

LeMMA 5.4. Let $w \in A_{p}$ and $\alpha \in \mathbb{C}$. The operator $T\left(\varphi_{\alpha}\right)$ is invertible on $\ell^{p}(w)$ if and only if $\operatorname{Re} \alpha \in I(w)$. 
Proof. Let $\operatorname{Re} \alpha \in I(w)$ and let $w_{\alpha}:=\left\{w_{n}(n+1)^{\operatorname{Re} \alpha}\right\}_{n=0}^{\infty}$. From the definition of $I(w)$ we infer $w_{\alpha} \in A_{p}$. Hence $T\left(\varphi_{-\alpha}\right) \in \mathcal{L}\left(\ell^{p}\left(w_{\alpha}\right)\right)$ by $(7)$. Because of (14), we have $M_{\alpha} \in \mathcal{L}\left(\ell^{p}\left(w_{\alpha}\right), \ell^{p}(w)\right)$ and $M_{-\alpha} \in \mathcal{L}\left(\ell^{p}(w), \ell^{p}\left(w_{\alpha}\right)\right)$. Thus,

$$
A_{\alpha}=\Gamma_{\alpha} M_{\alpha} T\left(\varphi_{-\alpha}\right) M_{-\alpha} \in \mathcal{C}\left(\ell^{p}(w), \ell^{p}(w)\right)=\mathcal{L}\left(\ell^{p}(w)\right) .
$$

As $I(w) \subset(-1,1)$ by Theorem 2.1 , we deduce from Corollary 5.2 that $T\left(\varphi_{\alpha}\right)$ is invertible on $\ell^{p}(w)$ provided $\alpha \notin \mathbb{Z}$. If $\alpha \in \mathbb{Z}$, then the relation $\operatorname{Re} \alpha \in I(w)$ gives that $\alpha=0$, which implies that $T\left(\varphi_{\alpha}\right)=I$ is invertible.

Conversely, assume that $T\left(\varphi_{\alpha}\right)$ is invertible on $\ell^{p}(w)$ and denote the inverse by $B_{\alpha}$. Suppose first that $\operatorname{Re} \alpha \geq 0$. Then $\ell^{p}\left(w_{\alpha}\right)$ is continuously embedded in $\ell^{p}(w)$ and hence $M_{-\alpha} \in \mathcal{L}\left(\ell^{p}(w), \ell^{p}(w)\right)$. Put $w_{-\alpha}=$ $\left\{w_{n}(n+1)^{-\operatorname{Re} \alpha}\right\}_{n=0}^{\infty}$. By $(7)$ and $(14), T\left(\varphi_{-\alpha}\right) \in \mathcal{L}\left(\ell^{p}(w)\right)$ and $M_{\alpha} \in$ $\mathcal{L}\left(\ell^{p}(w), \ell^{p}\left(w_{-\alpha}\right)\right)$. Consequently,

$$
A_{\alpha}=\Gamma_{\alpha} M_{\alpha} T\left(\varphi_{-\alpha}\right) M_{-\alpha} \in \mathcal{L}\left(\ell^{p}(w), \ell^{p}\left(w_{-\alpha}\right)\right)
$$

If $x \in \ell^{p}(w)$ is finitely supported, then $T\left(\varphi_{\alpha}\right) B_{\alpha} x=x$, and Corollary 5.2 (we exclude the trivial case where $\alpha \in \mathbb{Z}$ ) in conjunction with (25) therefore implies that $A_{\alpha} x=A_{\alpha} T\left(\varphi_{\alpha}\right) B_{\alpha} x=B_{\alpha} x$. Hence, $A_{\alpha} \in \mathcal{L}\left(\ell^{p}(w)\right)$.

Rewriting (16) in the form $T\left(\varphi_{-\alpha}\right)=\Gamma_{\alpha}^{-1} M_{\alpha}^{-1} A_{\alpha} M_{-\alpha}^{-1}$ and taking into consideration that $M_{\alpha}^{-1} \in \mathcal{L}\left(\ell^{p}(w), \ell^{p}\left(w_{\alpha}\right)\right)$ and $M_{-\alpha}^{-1} \in \mathcal{L}\left(\ell^{p}\left(w_{\alpha}\right), \ell^{p}(w)\right)$ by (14), we arrive at the conclusion that $T\left(\varphi_{-\alpha}\right) \in \mathcal{L}\left(\ell^{p}\left(w_{\alpha}\right)\right)$. Now Theorem 5.3 shows that necessarily $w_{\alpha} \in A_{p}$, which means $\operatorname{Re} \alpha \in I(w)$.

The proof is analogous in the case $\operatorname{Re} \alpha \leq 0$.

LEMma 5.5. Let $w \in A_{p}$ and $\alpha \in \mathbb{C}$. We have

$$
\begin{aligned}
& \operatorname{Re} \alpha \geq 0 \Rightarrow T\left(\varphi_{\alpha}\right) \text { is injective on } \ell^{p}(w), \\
& \operatorname{Re} \alpha \leq 0 \Rightarrow T^{*}\left(\varphi_{\alpha}\right) \text { is injective on } \ell^{q}\left(w^{-1}\right) .
\end{aligned}
$$

Proof. Suppose $\operatorname{Re} \alpha \geq 0$. In the preceding proof we observed that then (25) holds. Hence, if $x \in \ell^{p}(w)$ and $T\left(\varphi_{\alpha}\right) x=0$, we deduce from Corollary 5.2 (if $\alpha \notin \mathbb{Z}$, otherwise things are clear) that $x=A_{\alpha} T\left(\varphi_{\alpha}\right) x$ is the zero sequence in $\ell^{p}\left(w_{-\alpha}\right)$ and thus $x=0$. This proves (26). The implication (27) follows from (26) and the equality $T^{*}\left(\varphi_{\alpha}\right)=T\left(\varphi_{-\bar{\alpha}}\right)$ where $\bar{\alpha}$ is the complex conjugate of $\alpha$.

We are now in a position to prove Theorem 2.3 for the operators $T\left(\varphi_{\alpha}\right)$. Recall that

$$
\left(\varphi_{\alpha}\right)_{\mu, \nu}=\varphi_{\alpha}(\mathbb{T} \backslash\{\tau\}) \cup \mathcal{H}\left(e^{\pi i \alpha}, e^{-\pi i \alpha} ; \mu, \nu\right) .
$$

Theorem 5.6. Let $w \in A_{p}$ and let $0<\mu \leq \nu<1$ be the indices of powerlikeness of $w$. Let further $\alpha \in \mathbb{C}$ and $\kappa \in \mathbb{Z}$. The following are equivalent: (i) $T\left(\varphi_{\alpha}\right)$ is Fredholm of index $\kappa$ on $\ell^{p}(w)$;

(ii) $\operatorname{Re} \alpha+\kappa \in I(w)$;

(iii) $0 \notin\left(\varphi_{\alpha}\right)_{\mu, \nu}$ and wind $\left(\varphi_{\alpha}\right)_{\mu, \nu}=-\kappa$.

Proof. The equivalence (ii) $\Leftrightarrow$ (iii) is plane geometry. We confine ourselves to the proof of the equivalence (i) $\Leftrightarrow$ (ii).

Suppose $\operatorname{Re} \alpha+\kappa \in I(w)$. Then

$$
T\left(\varphi_{\alpha}\right)= \begin{cases}T\left(\varphi_{-\kappa}\right) T\left(\varphi_{\alpha+\kappa}\right) & \text { if } \kappa \geq 0, \\ T\left(\varphi_{\alpha+\kappa}\right) T\left(\varphi_{-\kappa}\right) & \text { if } \kappa \leq 0 .\end{cases}
$$

Since $T\left(\varphi_{\alpha+\kappa}\right)$ is invertible due to Lemma 5.4 and $T\left(\varphi_{-\kappa}\right)$ is Fredholm of index $\kappa$, we see that $T\left(\varphi_{\alpha}\right)$ is Fredholm of index $\kappa$.

Conversely, suppose $T\left(\varphi_{\alpha}\right)$ is Fredholm of index $\kappa$. We have

$$
T\left(\varphi_{\alpha+\kappa}\right)= \begin{cases}T\left(\varphi_{\alpha}\right) T\left(\varphi_{\kappa}\right) & \text { if } \kappa \geq 0, \\ T\left(\varphi_{\kappa}\right) T\left(\varphi_{\alpha}\right) & \text { if } \kappa \leq 0,\end{cases}
$$

and because $T\left(\varphi_{\kappa}\right)$ is Fredholm of index $-\kappa$, we conclude that $T\left(\varphi_{\alpha+\kappa}\right)$ is Fredholm of index zero. Using (26) for $\operatorname{Re} \alpha+\kappa \geq 0$ and (27) for $\operatorname{Re} \alpha+\kappa \leq 0$, we see that $T\left(\varphi_{\alpha+\kappa}\right)$ must in fact be invertible. Lemma 5.4 therefore implies that $\operatorname{Re} \alpha+\kappa \in I(w)$.

6. Localization. In this section we use localization techniques in order to derive Theorem 2.3 from Theorem 5.6.

We first state the local principle of Gohberg and Krupnik. Let $\mathcal{A}$ be a (complex) Banach algebra with identity $e$ and let $\mathcal{T}$ be an arbitrary set. Suppose for each $\tau \in \mathcal{T}$ we are given a set $\mathcal{M}_{\tau} \subset \mathcal{A}$ such that $0 \notin \mathcal{M}_{\tau}$ and such that for every $f_{1}, f_{2} \in \mathcal{M}_{\tau}$ there is an $f \in \mathcal{M}_{\tau}$ satisfying $f_{j} f=f f_{j}=f$ $(j=1,2)$. Furthermore, suppose that every collection $\left\{f_{\tau}\right\}_{\tau \in T}$ of elements $f_{\tau} \in \mathcal{M}_{\tau}$ contains a finite subcollection $\left\{f_{\tau_{j}}\right\}$ such that $\sum f_{\tau_{j}}$ is invertible in $\mathcal{A}$. Put $\mathcal{M}=\bigcup_{\tau \in \tau} \mathcal{M}_{\tau}$ and let $\operatorname{Com} \mathcal{M}$ stand for the set of all $a \in \mathcal{A}$ which commute with every element in $\mathcal{M}$. Two elements $a, b \in \mathcal{A}$ are said to be $\mathcal{M}_{\tau}$-equivalent if $\inf _{f \in \mathcal{M}_{\tau}}\|(a-b) f\|=\inf _{f \in \mathcal{M}_{\tau}}\|f(a-b)\|=0$.

THEOREM 6.1 (Gohberg and Krupnik). If $a \in \operatorname{Com} \mathcal{M}$ and if for every $\tau \in \mathcal{T}$ there exists an invertible element $a_{\tau} \in \mathcal{A}$ such that a and $a_{\tau}$ are $\mathcal{M}_{\tau}$-equivalent, then a itself is invertible.

A proof is in [11, Vol. I, Section 5.1] and [3, Theorem 1.31].

We apply this theorem to the Calkin algebra $\mathcal{A}=\mathcal{L}\left(\ell^{p}(w)\right) / \mathcal{K}\left(\ell^{p}(w)\right)$. Throughout this section we assume that $w \in A_{p}$. For $A \in \mathcal{L}\left(\ell^{p}(w)\right)$, we abbreviate the coset $A+\mathcal{K}\left(\ell^{p}(w)\right)$ to $A^{\pi}$. Let $\mathcal{T}=\mathbb{T}$, and for $\tau \in \mathbb{T}$, define $\mathcal{N}_{\tau}$ as the set of all functions $f \in C^{1}(\mathbb{T})$ with the following properties: $0 \leq f \leq 1, f=1$ in some open neighborhood $U_{f}$ of $\tau, f=0$ outside some open neighborhood $W_{f}$ of $\tau, f$ is monotone on $W_{f} \backslash U_{f}$. Clearly, 
$\|f\|_{\infty}=1$ and $\mathrm{V}(f)=2$ for every $f \in \mathcal{N}_{\tau}$. Inequality (7) therefore shows that $T^{\pi}(f) \in \mathcal{A}$ for all $f \in \mathcal{N}_{\tau}$. Put $\mathcal{M}_{\tau}=\left\{T^{\pi}(f): f \in \mathcal{N}_{\tau}\right\}$. To prove that the family $\left\{\mathcal{M}_{\tau}\right\}_{\tau \in \mathbb{T}}$ meets the assumptions made above, we need a few auxiliary results.

Let $\mathcal{P}$ be the collection of all trigonometric polynomials on $\mathbb{T}$. If $a \in \mathcal{P}$, then $L(a)$ is a band matrix and hence $\mathcal{P} \subset M_{p, w}$. We denote the closure of $\mathcal{P}$ in $M_{p, w}$ by $C_{p, w}$. Notice that $C_{2}=C:=C(\mathbb{T})$.

LEMMA 6.2. We have $C \cap \mathrm{BV} \subset C_{p, w} \subset C$.

Proof. Recall that we assume $w \in A_{p}$.

The inclusion $C_{p, w} \subset C$ is immediate from (8). In order to prove the inclusion $C \cap \mathrm{BV} \subset C_{p, w}$, pick $a \in C \cap \mathrm{BV}$. One can show as in [13] or [3, Lemma 2.44] that the Fejér-Cesàro means $\sigma_{n} a$ of any $a \in M_{p, w}$ satisfy the inequality

$$
\left\|\sigma_{n} a\right\|_{p, w} \leq\|a\|_{p, w}
$$

By Theorem 3.1, $\widetilde{w}:=w^{r} \in A_{p}$ for some $r>1$ sufficiently close to 1 . Since $w=\widetilde{w}^{1-\gamma} 1^{\gamma}$ for some $\gamma \in(0,1)$, the Stein-Weiss interpolation theorem gives

and (28) together with (7) implies that

$$
\left\|a-\sigma_{n} a\right\|_{p ; w} \leq\left\|a-\sigma_{n} a\right\|_{p, \tilde{w}}^{1-\gamma}\left\|a-\sigma_{n} a\right\|_{p}^{\gamma},
$$

$$
\left\|a-\sigma_{n} a\right\|_{p, \tilde{w}} \leq 2\|a\|_{p, \tilde{w}} \leq 2 C_{p, \tilde{w}}\left(\|a\|_{\infty}+V(a)\right) .
$$

Thus, it suffices to prove that $\left\|a-\sigma_{n} a\right\|_{p} \rightarrow 0$. It is clear that $\left\|a-\sigma_{n} a\right\|_{2}=$ $\left\|a-\sigma_{n} a\right\|_{\infty} \rightarrow 0$. If $p \neq 2$, we can have recourse to the Riesz-Thorin interpolation theorem to obtain

$$
\left\|a-\sigma_{n} a\right\|_{p} \leq\left\|a-\sigma_{n} a\right\|_{\tilde{p}}^{1-\delta}\left\|a-\sigma_{n} a\right\|_{2}^{\delta}
$$

for some $\tilde{p} \in(1,2) \cup(2, \infty)$ and some $\delta \in(0,1)$. Since, again by $(28)$ and $(7)$,

$$
\left\|a-\sigma_{n} a\right\|_{\tilde{p}} \leq 2\|a\|_{\tilde{p}} \leq 2 C_{\tilde{p}}\left(\|a\|_{\infty}+V(a)\right)
$$

it follows that $\left\|a-\sigma_{n} a\right\|_{p} \rightarrow 0$.

Lemma 6.3. If $a \in M_{p, w}$ and $f \in C_{p, w}$, then $T^{\pi}(a f)=T^{\pi}(a) T^{\pi}(f)=$ $T^{\pi}(f) T^{\pi}(a)$

Proof. We have $T(a f)=T(a) T(f)+H(a) \widetilde{H}(f)$ where $H(a)$ and $\tilde{H}(f)$ are the Hankel matrices

$$
H(a)=\left(a_{j+k+1}\right)_{j, k=0}^{\infty}, \quad \widetilde{H}(f)=\left(f_{-j-k-1}\right)_{j, k=0}^{\infty}
$$

(see, e.g., [3, Proposition 2.14]). Since $H(a)$ is a part of $L(a)$, we see that $H(a)$ induces a bounded operator on $\ell^{p}(w)$. (Recall that we assumed $w \in$ $A_{p}$.) The operator $\widetilde{H}\left(f_{n}\right)$ has finite rank for $f_{n} \in \mathcal{P}$. As $f \in C_{p, w}$ is the
$M_{p, w}$-limit of functions $f_{n} \in \mathcal{P}$ and

$$
\left\|\widetilde{H}(f)-\widetilde{H}\left(f_{n}\right)\right\|=\left\|\widetilde{H}\left(f-f_{n}\right)\right\| \leq\left\|f-f_{n}\right\|_{p, w},
$$

it results that $\widetilde{H}(f)$ is compact on $\ell^{p}(w)$. This proves $T^{\pi}(a f)=T^{\pi}(a) T^{\pi}(f)$.

The equality $T^{\pi}(f a)=T^{\pi}(f) T^{\pi}(a)$ can be verified analogously.

We can now prove that the sets $\mathcal{M}_{\tau}$ have the desired properties. Clearly, $0 \notin \mathcal{M}_{\tau}$. For $T^{\pi}\left(f_{1}\right), T^{\pi}\left(f_{2}\right) \in \mathcal{M}_{\tau}$, we have $f_{j} f=f f_{j}=f$ whenever $f \in \mathcal{N}_{\tau}$ has sufficiently small support. Lemmas 6.2 and 6.3 then give

$$
T^{\pi}\left(f_{j}\right) T^{\pi}(f)=T^{\pi}(f) T^{\pi}\left(f_{j}\right)=T^{\pi}(f) \text {. }
$$

Now let $\left\{T^{\pi}\left(f_{\tau}\right)\right\}_{\tau \in \mathbb{T}}$ be a collection of elements $T^{\pi}\left(f_{\tau}\right) \in \mathcal{M}_{\tau}$. As $\mathbb{T}$ is compact, there is a finite subcollection $\left\{f_{\tau_{j}}\right\}_{j=1}^{m}$ such that

$$
g:=f_{\tau_{1}}+\ldots+f_{\tau_{m}} \geq \varepsilon>0
$$

Since $g^{-1} \in C^{1}(\mathbb{T}) \subset C \cap \mathrm{BV}$, we deduce from Lemmas 6.2 and 6.3 that

$$
T^{\pi}\left(g^{-1}\right) T^{\pi}(g)=T^{\pi}(g) T^{\pi}\left(g^{-1}\right)=T^{\pi}(1)
$$

which shows that $T^{\pi}(g)$ is invertible.

LEMMA 6.4. Let $\tau \in \mathbb{T}$. If $a, b \in \mathrm{PC}_{p, w}$ and $a(\tau \pm 0)=b(\tau \pm 0)$, then $T^{\pi}(a)$ and $T^{\pi}(b)$ are $\mathcal{M}_{\tau}$-equivalent.

Proof. By the definition of $\mathrm{PC}_{p, w}$, there are $a_{n}, b_{n} \in \mathrm{BV}$ such that $\left\|a-a_{n}\right\|_{p, w} \rightarrow 0$ and $\left\|b-b_{n}\right\|_{p, w} \rightarrow 0$. We can clearly assume that $a_{n}(\tau \pm 0)=$ $a(\tau \pm 0)$ and $b_{n}(\tau \pm 0)=b(\tau \pm 0)$. From the definition of $\mathcal{M}_{\tau}$-equivalence we see that if $T^{\pi}\left(a_{n}\right)$ and $T^{\pi}\left(b_{n}\right)$ are $\mathcal{M}_{\tau}$-equivalent for all $n$, then $T^{\pi}(a)$ and $T^{\pi}(b)$ are $\mathcal{M}_{\tau}$-equivalent as well. Hence, we may assume without loss of generality that $a, b \in \mathrm{BV}$.

By Lemmas 6.2 and 6.3,

$$
\left\|\left(T^{\pi}(a)-T^{\pi}(b)\right) T^{\pi}(f)\right\|=\left\|T^{\pi}(f)\left(T^{\pi}(a)-T^{\pi}(b)\right)\right\| \leq\|(a-b) f\|_{p, w}
$$

for all $f \in \mathcal{N}_{\tau}$. Evidently, there are $f_{n} \in \mathcal{N}_{\tau}$ such that

$$
\left\|(a-b) f_{n}\right\|_{2}=\left\|(a-b) f_{n}\right\|_{\infty} \rightarrow 0
$$

Proceeding as in the proof of Lemma 6.2, we obtain $\|(a-b) f\|_{p, w} \rightarrow 0$.

Here is Theorem 2.3 for continuous symbols.

THEOREM 6.5. Let $a \in C_{p, w}$. The operator $T(a)$ is Fredholm on $\ell^{p}(w)$ if and only if a has no zeros on $\mathbb{T}$. In that case

$$
\text { Ind } T(a)=- \text { wind } a \text {. }
$$

Proof. Suppose $a$ has no zeros on $\mathbb{T}$. Lemma 6.3 tells us that $T^{\pi}(a) \in$ Com $\mathcal{M}$. By Lemma 6.4, $T^{\pi}(a)$ is $\mathcal{M}_{\tau}$-equivalent to $a(\tau) T^{\pi}(1)$ for each $\tau \in \mathbb{T}$, and since $a(\tau) T^{\pi}(1)$ has the inverse $(1 / a(\tau)) T^{\pi}(1)$, we deduce from Theorem 6.1 that $T^{\pi}(a)$ is invertible, i.e., that $T(a)$ is Fredholm. 
We now prove the index formula. So suppose $a$ has no zeros on $\mathbb{T}$. For any $b \in C_{p, w}, T(b)$ is Fredholm and Ind $T(b)=\operatorname{Ind} T(a)$ whenever $\|a-b\|_{p, w}$ is sufficiently small. By the definition of $C_{p, w}$, we may choose such a $b \in \mathcal{P}$, and clearly, we may assume that $b$ does not vanish and wind $b=$ wind $a$. We can write

$$
b(z)=b_{-}(z) z^{\text {wind } b} b_{+}(z) \quad(z \in \mathbb{C} \backslash\{0\})
$$

with $b_{ \pm} \in \mathcal{P}$ such that $b_{-}(z) \neq 0$ for $1 \leq|z| \leq \infty$ and $b_{+}(z) \neq 0$ for $|z| \leq 1$. It follows that $T(b)=T\left(b_{-}\right) T\left(t^{\text {wind } b}\right) T\left(b_{+}\right)$, and since $T\left(b_{ \pm}\right)$have the inverses $T\left(b_{ \pm}^{-1}\right)$, we get Ind $T(b)=\operatorname{Ind} T\left(t^{\text {wind } b}\right)=-$ wind $b$. Consequently, Ind $T(a)=$ Ind $T(b)=-$ wind $b=-$ wind $a$, which completes the proof of the index formula.

Finally, assume $a(\tau)=0$ for some $\tau \in \mathbb{T}$ but $T^{\pi}(a)$ is invertible. Set $b(t)=t-\tau$. By Lemma 6.4, $T^{\pi}(b)$ is $\mathcal{M}_{t}$-equivalent to the invertible element $T^{\pi}(a)$ at $t=\tau$ and $\mathcal{M}_{t}$-equivalent to the invertible element $(t-\tau) T^{\pi}(1)$ at $t \in \mathbb{T} \backslash\{\tau\}$. As $T^{\pi}(b) \in$ Com $\mathcal{M}$ by Lemma 6.3, we deduce from Theorem 6.1 that $T(b)$ must be Fredholm. It follows that $T(t-\zeta)$ is Fredholm of the same index as $T(b)$ for all $\zeta$ in some open neighborhood $U \subset \mathbb{C}$ of $\tau$. However, from the index formula already proved we see that there are $\zeta_{1}, \zeta_{2} \in U$ with Ind $T\left(t-\zeta_{1}\right)=0$ but $\operatorname{Ind} T\left(t-\zeta_{2}\right)=-1$. This contradiction proves that $a$ cannot have zeros on $\mathbb{T}$ if $T(a)$ is Fredholm.

Proof of Theorem 2.3. Lemmas 6.2 and 6.3 imply that $T^{\pi}(a) \in \operatorname{Com} \mathcal{M}$. We can therefore make use of Theorem 6.1.

Suppose $0 \notin a_{\mu, \nu}$. Then $a(\tau \pm 0) \neq 0$ for every $\tau \in \mathbb{T}$, and we can find $\gamma_{\tau} \in \mathbb{C}$ and $d_{\tau} \in \mathbb{C} \backslash\{0\}$ such that

$$
\begin{array}{ll}
\text { (29) } & a(\tau-0)=d_{\tau} e^{\pi i \gamma_{\tau}}=d_{\tau} \varphi_{\gamma_{\tau}, \tau}(\tau-0), \\
(30) & a(\tau+0)=d_{\tau} e^{-\pi i \gamma_{\tau}}=d_{\tau} \varphi_{\gamma_{\tau}, \tau}(\tau+0) .
\end{array}
$$

By Lemma $6.4, T^{\pi}(a)$ is $\mathcal{M}_{\tau}$-equivalent to $d_{\tau} T^{\pi}\left(\varphi_{\gamma_{r}, \tau}\right)$. From Theorem 5.6 we know that $d_{\tau} T^{\pi}\left(\varphi_{\gamma_{\tau}, \tau}\right)$ is invertible, and hence Theorem 6.1 yields the invertibility of $T^{\pi}(a)$.

Conversely, suppose $T^{\pi}(a)$ is invertible. We claim that then $a(\tau \pm 0) \neq 0$ for all $\tau \in \mathbb{T}$. Indeed, assume there is a $\tau \in \mathbb{T}$ such that $a(\tau-0)=0$ or $a(\tau+0)=0$. Since $a$ has at most countably many jumps and since invertibility of $T^{\pi}(a)$ is stable under small perturbations, we can assume that $a$ vanishes at a point $\tau$ at which it is continuous and that $T^{\pi}(a)$ is invertible. As in the proof of Theorem 6.5, this implies that $T^{\pi}(t-\tau)$ is invertible, which was shown not to be invertible. Thus, we have proved our claim.

Once $a(\tau \pm 0) \neq 0$, there are $\gamma_{\tau} \in \mathbb{C}$ and $d_{\tau} \in \mathbb{C} \backslash\{0\}$ satisfying (29), (30). Since $T^{\pi}\left(\varphi_{\gamma_{\tau}, \gamma}\right)$ is $\mathcal{M}_{t}$ equivalent to the invertible element $d_{\tau}^{-1} T^{\pi}(a)$ at $t=\tau$ and to the invertible element $\varphi_{\gamma_{\tau}, \tau}(t) T^{\pi}(1)$ at $t \in \mathbb{T} \backslash\{\tau\}$, Theorem 6.1 can be used to conclude that $T\left(\varphi_{\gamma_{\tau}, \tau}\right)$ is Fredholm. From Theorem 5.6 and (29), (30) we then deduce that

$$
0 \notin \mathcal{H}(a(\tau-0), a(\tau+0) ; \mu, \nu) .
$$

We are left with the index formula. Suppose for a moment that $a$ has only a finite number of jumps, at $\tau_{1}, \ldots, \tau_{m}$, say, and that $a \in \mathrm{BV}$. Then we can write $a=\varphi_{\gamma_{1}, \tau_{1}} \varphi_{\gamma_{2}, \tau_{2}} \ldots \varphi_{\gamma_{m}, \tau_{m}} c$ with appropriate $\gamma_{1}, \ldots, \gamma_{m} \in \mathbb{C}$ and a function $c$ in $C \cap$ BV. One can show as in [3, Proposition 6.29] that $T^{\pi}(a)=T^{\pi}\left(\varphi_{\gamma_{1}, \tau_{1}}\right) \ldots T^{\pi}\left(\varphi_{\gamma_{m}, \tau_{m}}\right) T^{\pi}(c)$, whence

$$
\operatorname{Ind} T\left(a_{i}\right)=\sum_{j=1}^{m} \operatorname{Ind} T\left(\varphi_{\gamma_{j}, \tau_{j}}\right)+\operatorname{Ind} T(c) \text {. }
$$

Theorems 5.6 and 6.5 therefore give

$$
\operatorname{Ind} T(a)=-\sum_{j=1}^{m} \operatorname{wind}\left(\varphi_{\gamma_{j}, \tau_{j}}\right)_{\mu, \nu}-\text { wind } c \text {. }
$$

Because

$$
\text { wind }(f g)_{\mu, \nu}=\text { wind } f_{\mu, \nu}+\text { wind } g_{\mu, \nu}
$$

whenever $f$ and $g$ have no common jumps, we arrive at formula (10).

Finally, for arbitrary $a \in \mathrm{PC}_{p, w}$ we can find $a_{n} \in \mathrm{BV}$ with only finitely many jumps such that $\left\|a-a_{n}\right\|_{\infty} \leq\left\|a-a_{n}\right\|_{p, w} \rightarrow 0$. As

$$
\operatorname{Ind} T\left(a_{n}\right) \rightarrow \operatorname{Ind} T(a), \quad \text { wind }\left(a_{n}\right)_{\mu, \nu} \rightarrow \text { wind } a_{\mu, \nu},
$$

and as (10) is true with $a$ replaced by $a_{n}$, we see that $(10)$ is valid for all $a \in \mathrm{PC}_{p, w}$.

7. Kernel and cokernel dimensions. In this section we prove Theorem 2.4 .

Recall that $w$ stands both for the weight $\left\{w_{n}\right\}_{n=0}^{\infty}$ on $\mathbb{Z}_{+}$and the weight $\left\{w_{|n|}\right\}_{n=-\infty}^{\infty}$ on $\mathbb{Z}$.

LEMMA 7.1. If $w \in A_{p}$, then there is a constant $C \in(0, \infty)$ depending only on $p$ and $w$ such that $w_{n+k} / w_{n} \leq C(|k|+1)$ for all $n, k \in \mathbb{Z}$.

Proof. The shift operator $U^{k}$ defined on $\ell^{p}(\mathbb{Z}, w)$ by $\left(U^{k} f\right)_{n}=f_{n-k}$ is nothing but the Laurent operator generated by $a(t)=t^{k}$, and (7) therefore implies that $\left\|U^{k}\right\| \leq C(|k|+1)$ with some constant $C \in(0, \infty)$ that depends only on $p$ and $w$. Letting $e_{n}$ be the $n$th element of the standard basis of $\ell^{p}(\mathbb{Z}, w)$, we obtain

$$
w_{n+k}^{p}=\left\|U^{k} e_{n}\right\|^{p} \leq C^{p}(|k|+1)^{p}\left\|e_{n}\right\|^{p}=C^{p}(|k|+1)^{p} w_{n}^{p} .
$$


The convolution $f * g$ of two sequences $f=\left\{f_{n}\right\}_{n \in \mathbb{Z}}$ and $g=\left\{g_{n}\right\}_{n \in \mathbb{Z}}$ is formally defined by

$$
(f * g)_{n}=\sum_{k \in \mathbb{Z}} f_{k} g_{n-k} \quad(n \in \mathbb{Z}) .
$$

LEMMA 7.2. Let $w \in A_{p}$. If $y \in \ell^{q}\left(\mathbb{Z}, w^{-1}\right)$ and $x \in \ell^{p}(\mathbb{Z}, w)$ then, for every $n \in \mathbb{Z}$,

$$
\left|(y * x)_{n}\right| \leq C(|n|+1)\|y\|_{\ell^{q}\left(\mathbb{Z}, w^{-1}\right)}\|x\|_{\ell^{p}(\mathbb{Z}, w)}
$$

where $C$ is the constant from Lemma 7.1.

Proof. We have

$$
\begin{aligned}
\left|(y * x)_{n}\right| & =\left|\sum_{k \in \mathbb{Z}} y_{k} x_{n-k}\right| \leq\left(\sum_{k \in \mathbb{Z}}\left|y_{k}\right|^{q} w_{k}^{-q}\right)^{1 / q}\left(\sum_{k \in \mathbb{Z}}\left|x_{n-k}\right|^{p} w_{k}^{p}\right)^{1 / p} \\
& =\left(\sum_{k \in \mathbb{Z}}\left|y_{k}\right|^{q} w_{k}^{-q}\right)^{1 / q}\left(\sum_{l \in \mathbb{Z}}\left|x_{l}\right|^{p} w_{n-l}^{p}\right)^{1 / p}
\end{aligned}
$$

and since $w_{n-l} / w_{l}=w_{l-n} / w_{l} \leq C(|n|+1)$ by Lemma 7.1, we arrive at the assertion.

Formula (9) shows that $M_{p, w}=M_{q, w^{-1}}$ and that $\|a\|_{p, w}=\|a\|_{q, w^{-1}}$.

LEMmA 7.3. If $w \in A_{p}, a \in M_{p, w}, y \in \ell^{q}\left(\mathbb{Z}, w^{-1}\right), x \in \ell^{p}(\mathbb{Z}, w)$, then $(y * a) * x$ and $y *(a * x)$ are well defined sequences and

$$
[(y * a) * x]_{n}=[y *(a * x)]_{n} \quad \text { for all } n \in \mathbb{Z} .
$$

Proof. From Lemma 7.2 we infer that

$$
\begin{aligned}
\left|[(y * a) * x]_{n}\right| & \leq C(|n|+1)\|y * a\|_{\ell^{q}\left(\mathbb{Z}, w^{-1}\right)}\|x\|_{\ell^{p}(\mathbb{Z}, w)} \\
& \leq C(|n|+1)\|a\|_{q, w^{-1}}\|y\|_{\ell^{q}\left(\mathbb{Z}, w^{-1}\right)}\|x\|_{\ell^{p}(\mathbb{Z}, w)} \\
\left|[y *(a * x)]_{n}\right| & \leq C(|n|+1)\|y\|_{\ell^{q}\left(\mathbb{Z}, w^{-1}\right)}\|a * x\|_{\ell^{p}(\mathbb{Z}, w)} \\
& \leq C(|n|+1)\|y\|_{\ell^{q}\left(\mathbb{Z}, w^{-1}\right)}\|a\|_{p, w}\|x\|_{\ell^{p}(\mathbb{Z}, w)} .
\end{aligned}
$$

The equalities (31) are easily verified in case $y$ and $x$ have finite supports. By virtue of (32) and (33), these equalities hold for all $y \in \ell^{q}\left(\mathbb{Z}, w^{-1}\right)$ and $x \in \ell^{p}(\mathbb{Z}, w)$.

A classical result by Coburn [5] and Simonenko [16] states that if $a \in$ $L^{\infty} \backslash\{0\}$, then $T(a)$ has a trivial kernel or a dense range on $\ell^{2}$. Duduchava [6], [8] proved this result for $a \in M_{p} \backslash\{0\}$ and $T(a)$ on $\ell^{p}$ (see also [3, Theorems 2.38 and 6.6$]$ ). Under the additional assumption that $a$ is invertible in $M_{p, w}$, we are able to extend the result to operators on $\ell^{p}(w)$.

THEOREM 7.4. Let $w \in A_{p}$ and $a \in M_{p, w}$. If $a$ is invertible in the Banach algebra $M_{p, w}$, then $T(a)$ has a trivial kernel or a dense range on
$\ell^{p}(w)$.
Proof. Assume the contrary, that is, assume there are $x_{+} \in \ell^{p}(w) \backslash\{0\}$ and $y_{+} \in \ell^{q}\left(w^{-1}\right) \backslash\{0\}$ such that $T(a) x_{+}=0$ and $T(\bar{a}) y_{+}=0$. Extend $x_{+}$ and $y_{+}$by zero to all of $\mathbb{Z}$. It follows that and

$$
L(a) x_{+}=a * x_{+}=: x_{-}, \quad x_{-} \in \ell^{p}(\mathbb{Z}, w), \quad\left(x_{-}\right)_{n}=0 \quad \text { for } n \geq 0,
$$

$L(\bar{a}) y_{+}=V a * y_{+}=: y_{-}, \quad y_{-} \in \ell^{q}\left(\mathbb{Z}, w^{-1}\right), \quad\left(y_{-}\right)_{n}=0 \quad$ for $n \geq 0$, where $V$ is defined by $(V f)_{n}=\bar{f}_{-n}$. It is easily seen that $V(f * g)=V f * V g$.

Taking Lemma 7.3 into account, we get

$$
\begin{aligned}
V y_{-} * x_{+} & =V\left(V a * y_{+}\right) * x_{+}=\left(a * V y_{+}\right) * x_{+} \\
& =\left(V y_{+} * a\right) * x_{+}=V y_{+} *\left(a * x_{+}\right)=V y_{+} * x_{-}
\end{aligned}
$$

Because $\left(V y_{-} * x_{+}\right)_{n}=0$ for $n \leq 0$ and $\left(V y_{+} * x_{-}\right)_{n}=0$ for $n \geq 0$, we see that $\left(V y_{+} * x_{-}\right)_{n}=0$ for all $n \in \mathbb{Z}$. Consequently,

$$
\begin{aligned}
\left(\bar{y}_{+}\right)_{0}\left(x_{-}\right)_{-1} & =0, \\
\left(\bar{y}_{+}\right)_{0}\left(x_{-}\right)_{-2}+\left(\bar{y}_{+}\right)_{1}\left(x_{-}\right)_{-1} & =0,
\end{aligned}
$$$$
\left(\bar{y}_{+}\right)_{0}\left(x_{-}\right)_{-3}+\left(\bar{y}_{+}\right)_{1}\left(x_{-}\right)_{-2}+\left(\bar{y}_{+}\right)_{2}\left(x_{-}\right)_{-1}=0, \ldots
$$

Since $y_{+} \neq 0$, it results that $\left(x_{-}\right)_{-1}=\left(x_{-}\right)_{-2}=\left(x_{-}\right)_{-3}=\ldots=0$.

Hence $x_{-}=0$, which implies that $L(a) x_{+}=0$. As $L(a)$ has the inverse $L\left(a^{-1}\right)$, we arrive at the conclusion that $x_{+}=0$. This contradicts our assumption.

Proof of Theorem 2.4. Let $a \in \mathrm{PC}_{p, w}$ and suppose $T(a)$ is Fredholm of index $\kappa$. Then $a(\tau \pm 0) \neq 0$ for all $\tau \in \mathbb{T}$ by virtue of Theorem 2.3. In particular, $a$ is invertible in $L^{\infty}$. We claim that $a^{-1}$ belongs to $\mathrm{PC}_{p, w}$.

In order to prove the claim, we employ Theorem 6.1 with $\mathcal{A}=\mathrm{PC}_{p, w}$ and with $\left\{\mathcal{M}_{\tau}\right\}_{\tau \in \mathbb{T}}$ replaced by the family $\left\{\mathcal{N}_{\tau}\right\}_{\tau \in \mathbb{T}}$ introduced in Section 6 . Simple modifications of the proof of Lemma 6.4 show that $a, b \in \mathrm{PC}_{p, w}$ are $\mathcal{N}_{\tau}$-equivalent if (and only if) $a(\tau \pm 0)=b(\tau \pm 0)$. Thus, with $\gamma_{\tau} \in \mathbb{C}$ and $d_{\tau} \in \mathbb{C} \backslash\{0\}$ given by (29) and (30), $a$ is $\mathcal{N}_{\tau}$-equivalent to $d_{\tau} \varphi_{\gamma_{\tau}, \tau}$. The inverse of $d_{\tau} \varphi_{\gamma_{\tau}, \tau}$ is $d_{\tau}^{-1} \varphi_{-\gamma_{\tau}, \tau}$ and therefore belongs to $\mathrm{PC}_{p, w}$. So Theorem 6.1 implies that $a$ is invertible in $\mathrm{PC}_{p, w}$, which completes the proof of our claim.

From Theorem 7.4 we now deduce that the (normally solvable) operator $T(a)$ is injective or surjective. This gives all assertions of Theorem 2.4 immediately.

\section{References}

[1] A. Böttcher and Yu. I. Karlovich, Carleson Curves, Muckenhoupt Weights, and Toeplitz Operators, Progr. Math. 154, Birkhäuser, Basel, 1997. 
[2] A. Böttcher and M. Seybold, Wackelsatz and Stechkin's inequality for discrete Muckenhoupt weights, preprint 99-7, TU Chemnitz, 1999.

[3] A. Böttcher and B. Silbermann, Analysis of Toeplitz Operators, AkademieVerlag, Berlin, 1989, and Springer, Berlin, 1990.

[4] A. Böttcher and I. Spitkovsky, Wiener-Hopf integral operators with $P C$ symbols on spaces with Muckenhoupt weight, Rev. Mat. Iberoamericana 9 (1993), 257-279.

[5] L. A. Coburn, Weyl's theorem for non-normal operators, Michigan Math. J. 13 (1966), 285-286.

[6] R. V. Duduchava, Discrete Wiener-Hopf equations in $\ell^{p}$ spaces with weight, Soobshch. Akad. Nauk Gruzin. SSR 67 (1972), 17-20 (in Russian).

[7] - On convolution integral operators with discontinuous symbols, Trudy Tbiliss. Mat. Inst. 50 (1975), 33-41 (in Russian).

[8] -, On discrete Wiener-Hopf equations, ibid., 42-59 (in Russian).

[9] -, Integral Equations in Convolution with Discontinuous Presymbols, Singular Integral Equations with Fixed Singularities, and Their Applications to Some Problems of Mechanics, Teubner, Leipzig 1979.

[10] J. García-Cuerva and J. L. Rubio de Francia, Weighted Norm Inequalities and Related Topics, North-Holland Math. Stud. 116, North-Holland, Amsterdam, 1985.

[11] I. Gohberg and N. Krupnik, One-Dimensional Linear Singular Integral Equations, Vols. I and II, Oper. Theory Adv. Appl. 53 and 54, Birkhäuser, Basel, 1992 (Russian original: Shtiintsa, Kishinev, 1973).

[12] R. Hunt, B. Muckenhoupt and R. Wheeden, Weighted norm inequalities for the conjugate function and Hilbert transform, Trans. Amer. Math. Soc. 176 (1973), $227-252$.

13] N. K. Nikol'skir̆, On spaces and algebras of Toeplitz matrices acting on $\ell^{p}$, Sibirsk. Mat, Zh. 7 (1966), 146-158 (in Russian).

[14] S. Roch and B. Silbermann, Algebras of convolution operators and their image in the Calkin algebra, report R-Math-05/90, Karl-Weierstrass-Inst. f. Math., Berlin, 1990.

[15] R. Schneider, Integral equations with piecewise continuous coefficients in the $L^{p}$ spaces with weight, J. Integral Equations 9 (1985), 135-152.

[16] I. B. Simonenko, Some general questions of the theory of the Riemann boundary value problem, Math. USSR-Izv. 2 (1968), 1091-1099.

[17] I. Spitkovsky, Singular integral operators with $P C$ symbols on the spaces with general weights, J. Funct. Anal. 105 (1992), 129-143.

[18] S. B. Stechkin, On bilinear forms, Dokl. Akad. Nauk SSSR 71 (1950), 237-240 (in Russian).

[19] J.-O. Strömberg and A. Torchinsky, Weighted Hardy Spaces, Lecture Notes in Math. 1381, Springer, Berlin, 1989

Fakultät für Mathematik

TU Chernnitz

D-09107 Chemnitz, Germany

E-mail: albrecht.boettcher@mathematik.tu-chemnitz.de matthias.seybold@mathematik.tu-chemnitz.de

\section{The stability of Markov operators on Polish spaces}

by

TOMASZ SZAREK (Katowice)

Abstract. A sufficient condition for the asymptotic stability of Markov operators acting on measures defined on Polish spaces is presented.

1. Introduction. We study Markov operators defined on a Polish space $X$. Our goal is to prove sufficient conditions for the asymptotic stability of such operators. The crucial point in proving stability is to show the existence of an invariant measure. When Markov operators are defined on a compact space, the proof of the existence goes as follows. First we construct a positive, invariant functional defined on the space of all continuous bounded functions $f: X \rightarrow \mathbb{R}$ and then using the Riesz representation theorem we define an invariant measure. This method was extended by A. Lasota and J. Yorke to the case when $X$ is a locally compact and $\sigma$-compact metric space [8]. When $X$ is a Polish space this idea breaks down, since a positive functional may not correspond to a measure. Therefore we base on the concept of tightness. The main idea taken from [8] is nonexpansiveness in the Fortet-Mourier distance. It is known (for details see $[1,6,7,8,9]$ ) that a broad spectrum of Markov processes do not increase the distance between measures transported by the corresponding transition operators. For such operators our results could be applied.

The organization of the paper is as follows. Section 2 contains some notation from the theory of Markov operators. In Section 3 we give some general conditions for asymptotic stability and discuss the condition for nonexpansiveness of $P$.

2. Preliminaries. Let $(X, \varrho)$ be a Polish space, i.e. a separable, complete metric space. Throughout this paper $B(x, r)$ stands for the open ball in $X$ with centre at $x$ and radius $r$. For every set $C \subset X$ we denote by $\operatorname{diam} C$

2000 Mathematics Subject Classification: Primary 60J05, 26A18; Secondary 60J20, 39B12.

Key words and phrases: Markoy operators, asymptotic stability. 